\title{
Probing order parameter structure in iron-based superconductors using vortices
}

\author{
Eugeniu Plamadeala, T. Pereg-Barnea, and Gil Refael \\ Department of Physics, California Institute of Technology, 1200 E. California Boulevard, MC114-36, Pasadena, California 91125, USA
}

(Received 2 December 2009; revised manuscript received 13 March 2010; published 13 April 2010)

\begin{abstract}
Impurities, inevitably present in all samples, induce elastic transitions between quasiparticle states on the contours of constant energy. These transitions may be seen in Fourier-transformed scanning tunneling spectroscopy experiments, sorted by their momentum transfer. In a superconductor, anomalous scattering in the pairing channel may be introduced by magnetic field. When a magnetic field is applied, vortices act as additional sources of scattering. These additional transitions may enhance or suppress the impurity-induced scattering. We find that the vortex contribution to the transitions is sensitive to the momentum-space structure of the pairing function. In the iron-based superconductors, there are both electron and hole pockets at different regions of the Brillouin zone. Scattering processes therefore represent intrapocket or interpocket transitions, depending on the momentum transfer in the process. In this work we show that while in a simple $s$-wave superconductor all transitions are enhanced by vortex scattering, in an $s_{ \pm}$superconductor only intrapocket transitions are affected. We suggest this effect as a probe for the existence of the sign change in the order parameter.
\end{abstract}

DOI: 10.1103/PhysRevB.81.134513

PACS number(s): 74.20.Rp, 74.25.Jb, 74.70.-b

\section{INTRODUCTION}

In January 2008, Kamihara ${ }^{1}$ announced the discovery of superconductivity in $\mathrm{La}\left[\mathrm{O}_{1-x} \mathrm{~F}_{x}\right]$ FeAs and shortly after more compounds of the iron-based superconductors (FeSC) family were discovered. This family shares a number of important characteristics with the high- $T_{c}$ cuprates such as the layered structure and the proximity of the superconducting phase to a magnetic one. Given these similarities it is natural to ask whether the FeSC are conventional or unconventional. By "conventional" it is usually meant that the pairing mechanism is based on the interaction of fermions and phonons with a rotationally symmetric order parameter (OP), as described by the BCS theory. ${ }^{2}$ An "unconventional" superconductor may result from any other mechanism and its OP would have a nontrivial structure. This question is not easily answered since one cannot probe the underlying state, i.e., the state of the system without the pairing instability. A related question seems to be an easier starting point; what is the structure of the pairing function? In particular, we would like to be able to distinguish between a simple $s$-wave order parameter and other more complex OPs. A simple $s$-wave would very likely deem the FeSC family conventional and may restrict $T_{c}$. Any other result will suggest an unconventional pairing mechanism involving electric, magnetic, and/or lattice interactions.

The purpose of this paper is to propose an experiment to distinguish between two prominent candidates for the order parameter in the iron-based superconductors. As we discuss below, this may prove to be a difficult task. Armed with the experience of identifying the $d$-wave OP in the cuprates ${ }^{3,4}$ and state-of-the-art probes we are in a good position to distinguish between a simple $s$-wave structure and higher angular momentum OPs. The distinction is usually made through the observation (or lack) of nodal quasiparticles-lowenergy excitations that reside in the vicinity of the intersection between the Fermi surface (FS) and the OP nodal lines. Such nodal quasiparticles may be seen in thermodynamic properties such as transport or NMR relaxation rate. In the FeSCs, early NMR/nuclear quadrupole resonance experiments have reported the absence of coherence peaks and a power-law behavior of $1 / T_{1} T$ as a function of temperature, which have been formerly related to the nodal quasiparticles $^{5-9}$ However, it seems that these facts alone do not necessarily imply gapless excitations when more than one band is involved. ${ }^{10}$ Other experiments such as angle resolved photoemission (ARPES) ${ }^{11-13}$ microwave penetration depth, ${ }^{14,15}$ and others report nodeless gaps. To date, it seems that apart from LaFePO which has a $d$-wave order parameter $^{16}$ other compounds have nodeless OPs.

Based on the above findings we choose to focus on OPs with $s$-wave symmetry (in the lattice this would be a discrete rotational symmetry). The FeSC has five electrons in the $d$ shell of the iron and its low-energy band structure is composed of two concentric hole pockets around the Brillouinzone center (the $\Gamma$ point) and two electron pockets around $(0, \pi)$ and $(\pi, 0)$ (the $\mathrm{M}$ points). ${ }^{17}$ It is therefore possible that the order parameter changes its magnitude and phase between these two bands. In this work, we propose an experiment to distinguish between a simple $s$ wave, which does not change sign on or between the bands and the so-called $s_{ \pm}$ OP. The latter order parameter can be roughly sketched as a single function of momentum, $\Delta_{+}(\mathbf{k})=\Delta_{0} \cos \left(k_{x}\right) \cos \left(k_{y}\right)$ (Ref. 18) which has line nodes between the electron and hole pockets. It therefore gaps both Fermi surfaces and does not allow for nodal excitations. However, the sign of the order parameter changes from one pocket to the other. We focus on this OP since it arises from several microscopic models such as extended $t$ - $J$ model, ${ }^{19}$ FS nesting, exchange interactions, ${ }^{18,20}$ an interaction-induced density wave, ${ }^{21}$ and functional renormalization group of a strongly interacting lattice model. ${ }^{22}$

The $s_{ \pm}$order parameter poses a challenge for the experimental probes. Since both Fermi pockets are fully gapped, experiments which are mainly sensitive to the spectrum (ARPES, NMR, etc.) are incapable of detecting its sign change. On the other hand, a phase-sensitive probe such as 
the one devised by Tsuei and Kirtley ${ }^{3}$ for the cuprates is not easily achieved since the OP sign depends on the amplitude of the momentum rather than its direction. ${ }^{23,24} \mathrm{~A}$ natural route to explore is then the effect of intrinsic or induced scattering processes. We show below how intrapocket and interpocket scattering processes may be identified in Fouriertransformed (FT) scanning tunneling spectroscopy experiments. The scattering probability is sensitive to both the sign and magnitude of the OP. The response of these transitions to vortex scattering may reveal the sign difference between the initial and final states and allow the distinction between a simple $s$-wave and an $s_{ \pm}$OP.

This paper is organized as follows. In the next section we sketch the suggested experiment and its interpretations; in Sec. III we describe our minimal two-band model framework $^{17}$ and calculate the local-density-of-states (LDOS) modulations which arise from both impurity and vortex scattering ${ }^{25}$ and in Sec. IV we present and discuss our results.

\section{PROPOSED EXPERIMENT}

Spatial modulations in the LDOS are a signature of disorder. We model the disorder as a pointlike potential (impurity or vortex) and perform the Born approximation. When the LDOS modulations are measured at energy $\hbar \omega$ the relevant processes (elastically) take a quasiparticle from a momentum state $\mathbf{k}_{i}$ to a momentum state $\mathbf{k}_{f}$. The largest contribution to the LDOS modulations comes from the vicinity of the relevant contours of constant energy. When the LDOS is FT a feature appears at any momentum $\mathbf{q}=\mathbf{k}_{f}-\mathbf{k}_{i}$ which matches two points on the contours of constant energy. ${ }^{26}$ Each process's contribution is further weighted by quantummechanical considerations such as the phase space available for scattering ${ }^{27}$ and the scattering potential matrix elements between the initial and final wave functions. In superconductors, these matrix elements depend crucially on the amount of particle-hole mixing in the state, i.e., the Bogoliubov-de Gennes coherence factors ${ }^{28}$ which, in turn, depend on the magnitude and sign of the order parameter. As suggested by Zhang and $\mathrm{Hu},{ }^{29}$ one can recognize the fingerprint of the suggested $s_{ \pm}$OP in the quasiparticle interference maps. However, we suspect that the signature of the $s_{ \pm}$state might not be easy to identify in a disordered system. Our current suggestion builds on the quasiparticle interference maps idea and adds the magnetic field as a knob which will alter features in a way that can reveal the elusive $s_{ \pm}$state.

Our findings indicate that the relative intensity of the interpocket and intrapocket transitions will change dramatically when the magnetic field is turned on if the order parameter changes sign between pockets. The magnetic field creates vortices which are pinned to the impurity sites. These vortices act as scattering sources in the particle-particle (offdiagonal) channel. ${ }^{25}$ The contribution of the vortices to the LDOS modulations has features in the same momenta $\mathbf{q}$ as the impurity scattering (since $\mathbf{q}$ is determined by the contours of constant energy). The intensity, however, has a different dependence on the coherence factors. ${ }^{30}$ In fact, when the energy is tuned to the gap edge, the vortex scatter contribution, $\delta N_{v}$, is roughly

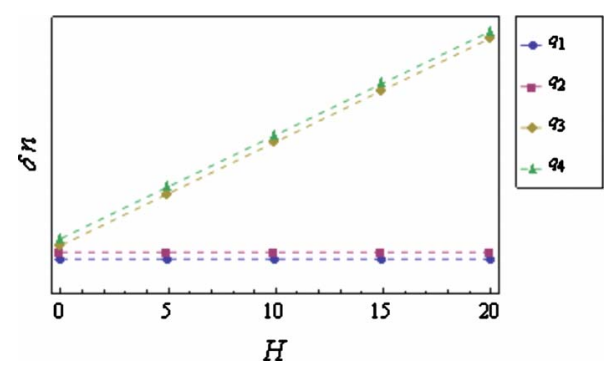

FIG. 1. (Color online) Schematic plot of feature intensity vs magnetic field. The intensity of the Fourier-transformed LDOS at momenta $\mathbf{q}_{i}$ (defined as momentum transfer in the scattering processes described in Fig. 2) as a function of magnetic field. When the magnetic field is applied the vortex-induced scattering is added to the impurity scattering. For intrapocket transitions $\left(\mathbf{q}_{3}, \mathbf{q}_{4}\right)$ the intensity is enhanced and for interpocket transitions $\left(\mathbf{q}_{1}, \mathbf{q}_{2}\right)$ it is not

$$
\delta N_{v}\left(\mathbf{q}, \omega \sim \Delta_{0}\right) \propto \omega\left[\Delta\left(\mathbf{k}_{i}\right)+\Delta\left(\mathbf{k}_{f}\right)\right]^{2} .
$$

This means that while intrapocket transitions are always enhanced, interpocket transitions are enhanced only if the sign of the OP is the same on both pockets. In the case of the $s_{ \pm}$ OP $\Delta\left(\mathbf{k}_{i}\right) \approx-\Delta\left(\mathbf{k}_{f}\right)$ for interpocket transitions which means no vortex contribution to those transitions. In other words-if the FeSC have an order parameter that changes sign between the electron and hole pockets then the application of magnetic field will affect the intensity of the Fouriertransformed LDOS at momenta $\mathbf{q}_{\text {intra }}$ which connect two points on the same pocket and will only weakly affect the intensity of features whose momentum $\mathbf{q}_{\text {inter }}$ connects two points on different pockets. The qualitative results of the suggested experiments are schematically plotted in Fig. 1 where the feature intensity is modified (or unchanged) when magnetic field is applied due to the addition of vortex scattering. In the next section we demonstrate this principle through a phenomenological, BCS-type model, based on a two-orbital band structure ${ }^{17}$ and the sign-changing $s_{ \pm}$OP.

\section{ANALYSIS}

In this section we review the two-band model for the FeSC (Ref. 17) and adopt it as the unperturbed Hamiltonian to describe the uniform system. We then consider quasiparticle scattering off impurities and vortices using the Born approximation.

\section{A. Two-band model}

The unperturbed Hamiltonian we use is given by

$$
\begin{gathered}
\mathcal{H}_{0}^{M F}=\sum_{k} \psi(\mathbf{k})^{\dagger} \hat{h}(\mathbf{k}) \psi(\mathbf{k}), \\
\hat{h}(\mathbf{k})=\hat{h}_{t}(\mathbf{k})+\Delta(\mathbf{k}) \sigma_{0} \otimes \tau_{1}, \\
\hat{h}_{t}(\mathbf{k})=\left\{\left[\epsilon_{+}(\mathbf{k})-\mu\right] \sigma_{0}+\epsilon_{-}(\mathbf{k}) \sigma_{3}+\epsilon_{x y}(\mathbf{k}) \sigma_{1}\right\} \otimes \tau_{3},
\end{gathered}
$$

where $\psi(\mathbf{k})^{\dagger}=\left(c_{\mathbf{k}, \uparrow}^{\dagger}, c_{-\mathbf{k}, \downarrow}, d_{\mathbf{k}, \uparrow}^{\dagger}, d_{-\mathbf{k}, \downarrow}\right)$ is a vector representing both the orbital and Nambu degrees of freedom. Here $c_{\mathbf{k}, \sigma}^{\dagger}$ 
creates an electron carrying momentum $\mathbf{k}$ and spin $\sigma$ in the " $d_{x z}$ " orbital and $d_{\mathbf{k}, \sigma}^{\dagger}$ creates and electron in the " $d_{y z}$ " orbital. The $\sigma$ Pauli matrices act in the orbital space and the $\tau$ Pauli matrices act in Nambu space. In what follows we consider the $s_{ \pm} \mathrm{OP}, \Delta(\mathbf{k})=\Delta_{0} \cos k_{x} \cos k_{y}$ unless otherwise stated. The band structure $\hat{h}_{t}$ is the result of hopping terms of the two orbits on nearest- and next-nearest bonds with the appropriate overlap amplitudes,

$$
\begin{gathered}
\epsilon_{+}(\mathbf{k})=-\left(t_{1}+t_{2}\right)\left(\cos k_{x}+\cos k_{y}\right)-4 t_{3} \cos k_{x} \cos k_{y}, \\
\epsilon_{-}(\mathbf{k})=-\left(t_{1}-t_{2}\right)\left(\cos k_{x}-\cos k_{y}\right), \\
\epsilon_{x y}(\mathbf{k})=-4 t_{4} \sin k_{x} \sin k_{y} .
\end{gathered}
$$

A realistic set of parameters is: $t_{1}=-1, t_{2}=1.3, t_{3}=t_{4}=$ $-0.85, \Delta_{0}=0.1$ and $\mu$ between 1.3 and 1.9 , where energy is measured in units of $\left|t_{1}\right| \cdot{ }^{17}$ The unperturbed, retarded Green's function $G^{0}(\mathbf{k}, \omega)=\left[(\omega+i \eta) \mathbb{I}_{4}-\hat{h}(\mathbf{k})\right]^{-1}$.

\section{B. Impurity and vortex scattering potential}

Next we consider a perturbation and its effect on the local density of states. The perturbing Hamiltonian is of the form

$$
\delta \mathcal{H}=\sum_{\mathbf{k}, b \mathbf{k}^{\prime}} d \mathbf{k} d \mathbf{k}^{\prime} \Psi_{\mathbf{k}}^{\dagger} V_{\mathbf{k}, \mathbf{k}^{\prime}} \Psi_{\mathbf{k}^{\prime}},
$$

where $V_{\mathbf{k}, \mathbf{k}^{\prime}}$ is a $4 \times 4$ matrix in the two-band Nambu space. Its matrix elements are general enough to describe on-site or hopping-like potential in both the particle-hole channel (charge/spin impurity) or the particle-particle channel (pairing perturbation).

In the Born approximation, the perturbed Green's function is given by

$$
\begin{gathered}
G\left(\mathbf{k}, \mathbf{k}^{\prime}, \omega\right)=G^{0}(\mathbf{k}, \omega) \delta_{\mathbf{k}, \mathbf{k}^{\prime}}+\delta G\left(\mathbf{k}, \mathbf{k}^{\prime} \omega\right), \\
\delta G\left(\mathbf{k}, \mathbf{k}^{\prime}, \omega\right)=G^{0}(\mathbf{k}, \omega) V_{\mathbf{k}, \mathbf{k}^{\prime}} G^{0}\left(\mathbf{k}^{\prime}, \omega\right) .
\end{gathered}
$$

As a result, the induced FT LDOS modulations are given by $^{29}$

$$
\begin{gathered}
\delta n(\mathbf{q}, \omega)=-\frac{1}{\pi} \operatorname{Im} \int \frac{d^{2} k}{(2 \pi)^{2}} \\
{\left[\delta G(\mathbf{k}, \mathbf{k}+\mathbf{q}, \omega)_{11}+\delta G(\mathbf{k}, \mathbf{k}+\mathbf{q}, \omega)_{33}\right] .}
\end{gathered}
$$

We classify the various potentials in Eq. (5) in the following way. In orbital space, a perturbation may be diagonal (such that it does not mix the $d_{x z}$ and the $d_{y z}$ orbits) or off-diagonal. We follow Ref. 29 and focus on the orbitally diagonal perturbation. Our conclusion does not depend on this choice. In the Nambu space diagonal disorder represents impurities that may couple to charge (nonmagnetic impurity, $\tau_{3}$ ) or spin (magnetic impurity, $\tau_{0}$ ). These two perturbations are closely related and we present here only the nonmagnetic case. Offdiagonal perturbations in Nambu space are related to pairing, we shall see below that the presence of a vortex takes this form.

A pointlike nonmagnetic impurity is simply described by

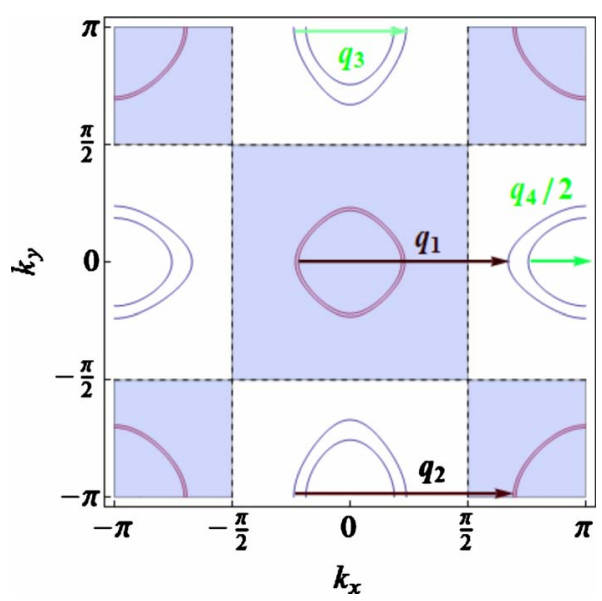

FIG. 2. (Color online) Contours of constant energy. The iron Brillouin zone with the contours of constant energy, $\omega=0.105$, right at the gap edge. The (red) contours around the $\Gamma$ points are the hole pockets and the (blue) contours around the $\mathrm{M}$ points are the electron pockets. The vectors $\mathbf{q}_{1}$ and $\mathbf{q}_{2}$ are interpocket transitions while $\mathbf{q}_{3}$ and $\mathbf{q}_{4}$ are intrapocket transitions in the hole and electron pockets, respectively. The nodal lines of the $s_{ \pm}$order parameter are marked by the horizontal and vertical dashed lines and the areas with a positive (negative) OP are shaded (unshaded).

$$
\delta \mathcal{H}=V_{0} \sum_{\sigma}\left[c_{\sigma}^{\dagger}\left(\mathbf{r}_{0}\right) c_{\sigma}\left(\mathbf{r}_{0}\right)+d_{\sigma}^{\dagger}\left(\mathbf{r}_{0}\right) d_{\sigma}\left(\mathbf{r}_{0}\right)\right]
$$

where $c_{\sigma}(\mathbf{r})$ represent the electron annihilation operators on site $\mathbf{r}$. We chose a perturbation which does not mix the two orbitals. In principle, this may not be the only contribution. However, previous studies have shown that the orbitally diagonal perturbation is the dominant one. ${ }^{29}$ In the language of the two-band Nambu space the perturbation potential is $V_{\mathbf{k}, \mathbf{k}^{\prime}}=V_{0} \sigma_{0} \otimes \tau_{3}$. This perturbation has been studied in Ref. 29 and we do not wish to repeat the analysis. Instead we draw the reader's attention to four scattering processes along the $x$ axis. They are denoted by their momentum-transfer vectors $\mathbf{q}_{1}, \mathbf{q}_{2}, \mathbf{q}_{3}$, and $\mathbf{q}_{4}$ in Fig. 2. Of these transitions $\mathbf{q}_{1}$ and $\mathbf{q}_{2}$ are interpocket transitions, and $\mathbf{q}_{3}$ and $\mathbf{q}_{4}$ are intrapocket. We can clearly see these transitions in the dashed (online red) curves in Fig. 3, in both panels. Moreover, one may notice that in the presence of a nonmagnetic impurity intrapocket and interpocket transitions have opposite signs of intensity in the $s_{ \pm}$scenario and the same sign in the simple $s$-wave case. This could have, in principle, served as a way to distinguish between the two OPs. However, this may be problematic because (i) scanning tunneling spectroscopy experiments are not sensitive to this $\operatorname{sign}^{31}$ and (ii) the magnetic scattering channel $\left(\tau_{0}\right)$ mixes in with the nonmagnetic $\left(\tau_{3}\right)$ even though the impurity has no magnetic moment when scattering is strong. ${ }^{32}$ We shall show below that the analysis that makes use of the vortex-induced scattering is free of these problems.

Let us consider a magnetically induced vortex. We simplify its effect by considering only the amplitude suppression of the order parameter near the vortex core. This simplification is crucial for our analysis; including the OP phase winding leads to technical complications which, at the moment, 

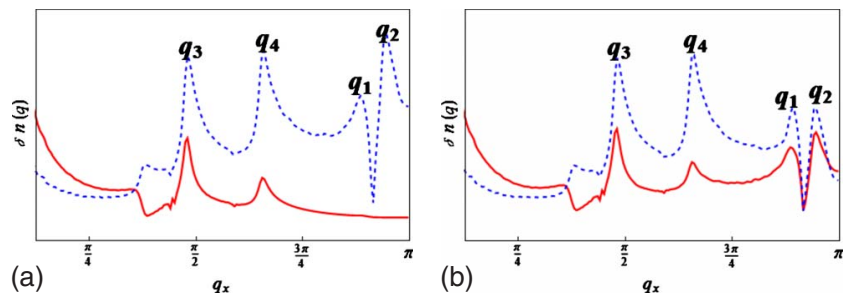

FIG. 3. (Color online) Quasiparticle interference cuts. The tunneling density-of-states modulations $\delta n(\mathbf{q})$, Eqs. (10) and (12)-(17), plotted along $q_{y}=0$ for $q_{x} \in(0, \pi)$. The transitions $\mathbf{q}_{i}$ are the same as in Fig. 2. Both plots are for the same parameters as in Fig. 2: $\mu=1.5, \omega=0.105$. In both pallets the solid (red) line represents vortex scattering and the dashed (blue) line is for impurity scattering. Both curves of pallet (a) were generated with the $s_{ \pm}$order parameter while, for comparison, the curves in pallet (b) was generated using the absolute value $\left|\Delta_{ \pm}(\mathbf{k})\right|$. The presence of peaks at $\mathbf{q}_{1}$ and $\mathbf{q}_{2}$ in these plots is a confirmation that the sign change in $s_{ \pm}$ plays a crucial role in their suppression in pallet (a).

we are unable to overcome. Nevertheless, the same simplification has been carried out for a $d$-wave superconductor and proved useful in explaining experimental results. ${ }^{25} \mathrm{We}$ consider a "pointlike vortex" by suppressing the OP amplitude only on four next-nearest neighbor links around a single site (labeled zero). Larger cores may be taken into account by adding up a few of these perturbations. The modification to the Hamiltonian caused by the vortex is described by

$$
\begin{aligned}
\delta \mathcal{H}= & \sum_{\delta} \delta \Delta\left[c_{\uparrow}\left(r_{0}\right) c_{\downarrow}\left(r_{0}+\hat{\delta}\right)\right. \\
& \left.-c_{\downarrow}\left(r_{0}\right) c_{\uparrow}\left(r_{0}+\hat{\delta}\right)+(c \rightarrow d)+\text { H.c. }\right],
\end{aligned}
$$

where $\hat{\delta}= \pm \hat{x} \pm \hat{y}$. In the two-band-Nambu basis we get

$$
V_{\mathbf{k k}^{\prime}}=4 \delta \Delta\left(\chi_{\mathbf{k}}+\chi_{\mathbf{k}^{\prime}}\right) \sigma_{0} \otimes \tau_{1},
$$

where $\chi_{\mathbf{k}}=\cos k_{x} \cos k_{y}=\Delta_{ \pm}(\mathbf{k}) / \Delta_{0}$ is the result of nextnearest-neighbor summation.

\section{FEATURE INTENSITIES}

\section{A. Intrapocket and interpocket scattering}

Next we turn to evaluating the induced modulations in the LDOS in both cases of scattering. In order to distinguish between intrapocket and interpocket scattering processes it is useful to work in the pocket (energy-band) basis rather than the orbital basis. To do this we transform the $4 \times 4$ matrices of the Green's functions and potentials through the unitary transformation,

$$
\begin{gathered}
U(\mathbf{k})=\left[\cos \left(\beta_{\mathbf{k}} / 2\right) \sigma_{0}-\sin \left(\beta_{\mathbf{k}} / 2\right) \sigma_{2}\right] \otimes \tau_{0} . \\
\beta_{\mathbf{k}}=\arctan \left[\frac{\epsilon_{x y}(\mathbf{k})}{\epsilon_{-}(\mathbf{k})}\right] .
\end{gathered}
$$

This transformation diagonalizes the kinetic part of the Hamiltonian: $\hat{h}_{t}$. We define the Green's function and the potential in the pocket basis by

$$
\begin{aligned}
\widetilde{G}^{0}(\mathbf{k}, \omega) & =U(\mathbf{k})^{-1} G^{0}(\mathbf{k}, \omega) U(\mathbf{k}), \\
\widetilde{V}_{\mathbf{k}, \mathbf{k}^{\prime}} & =U(\mathbf{k})^{-1} V_{\mathbf{k}, \mathbf{k}^{\prime}} U\left(\mathbf{k}^{\prime}\right) .
\end{aligned}
$$

Applying the above transformations to $\delta G\left(\mathbf{k}, \mathbf{k}^{\prime}, \omega\right)$ in the Born approximation [Eq. (5)] we find

$$
\begin{aligned}
& \left.\delta G(\mathbf{k}, \mathbf{k}+\mathbf{q}, \omega)\right|_{11+33}=V_{0}\left(\left[P_{1}(\mathbf{k}, \mathbf{q})+P_{2}(\mathbf{k}, \mathbf{q})\right]\right. \\
& \left.\quad \times \cos ^{2} \frac{\beta_{\mathbf{k}}-\beta_{\mathbf{k}+\mathbf{q}}}{2}+\left[Q_{1}(\mathbf{k}, \mathbf{q})+Q_{2}(\mathbf{k}, \mathbf{q})\right] \sin ^{2} \frac{\beta_{\mathbf{k}}-\beta_{\mathbf{k}+\mathbf{q}}}{2}\right) .
\end{aligned}
$$

In the above expression the sine and cosine functions are the result of the transformation. The "angle" represents the amount of orbital mixing in the bands. It is interesting to note that on the $\hat{x}$ and $\hat{y}$ axes the orbitals are not mixed (since $\epsilon_{x y}=0$ ). This prohibits interband on-shell transitions on the axis. However, intensity around the vectors $\mathbf{q}_{1}$ and $\mathbf{q}_{2}$ is still nonvanishing due to the contribution of close-by off-shell states. The functions $P_{1(2)}(\mathbf{k}, \mathbf{q})$ represent intrapocket transitions within the kinetic-energy band $\epsilon_{1 / 2}(\mathbf{k})$ $=\epsilon_{+}(\mathbf{k}) \pm \sqrt{\epsilon_{-}^{2}(\mathbf{k})+\epsilon_{x y}^{2}(\mathbf{k})}$ and the functions $Q_{1(2)}(\mathbf{k}, \mathbf{q})$ represent transitions from one band to another. In the case of a nonmagnetic impurity we find

$$
\begin{aligned}
& P_{1(2)}=\frac{\left[\omega+\epsilon_{1(2)}(\mathbf{k})\right]\left[\omega+\epsilon_{1(2)}(\mathbf{k}+\mathbf{q})\right]-\Delta(\mathbf{k}) \Delta(\mathbf{k}+\mathbf{q})}{\left[\omega^{2}-\epsilon_{1(2)}(\mathbf{k})^{2}-\Delta(\mathbf{k})^{2}+i \eta\right]\left[\omega^{2}-\epsilon_{1(2)}(\mathbf{k}+\mathbf{q})^{2}-\Delta(\mathbf{k}+\mathbf{q})^{2}+i \eta\right]}, \\
& Q_{1(2)}=\frac{\left[\omega+\epsilon_{1(2)}(\mathbf{k})\right]\left[\omega+\epsilon_{2(1)}(\mathbf{k}+\mathbf{q})\right]-\Delta(\mathbf{k}) \Delta(\mathbf{k}+\mathbf{q})}{\left[\omega^{2}-\epsilon_{1(2)}(\mathbf{k})^{2}-\Delta(\mathbf{k})^{2}+i \eta\right]\left[\omega^{2}-\epsilon_{2(1)}(\mathbf{k}+\mathbf{q})^{2}-\Delta(\mathbf{k}+\mathbf{q})^{2}+i \eta\right]} .
\end{aligned}
$$

By tuning the energy (or the external bias in the experiment) to the gap edge we may assume that the kinetic energy is roughly equal to the chemical potential such that $\epsilon_{i}(k) \sim 0$. This simplifies the functions $P$ and $Q$ to 


$$
P_{i}(\mathbf{k}, \mathbf{q}) \approx Q_{i}(\mathbf{k}, \mathbf{q}) \approx \frac{\omega^{2}-\Delta(\mathbf{k}) \Delta(\mathbf{k}+\mathbf{q})}{\left[\omega^{2}-\Delta(\mathbf{k})^{2}+i \eta\right]\left[\omega^{2}-\Delta(\mathbf{k}+\mathbf{q})^{2}+i \eta\right]} .
$$

Note that the imaginary part of the above expression (which contributes to the observed LDOS) is an odd function of $\omega$.

A similar derivation can be done for the vortex perturbation. Equation (12) remains the same except for the replacement of $V_{0}$ by $\delta \Delta$ and the functions $P$ and $Q$ are

$$
\begin{aligned}
& P_{1(2)}=\frac{\Delta(\mathbf{k})\left[\omega+\epsilon_{1(2)}(\mathbf{k}+\mathbf{q})\right]+\Delta(\mathbf{k}+\mathbf{q})\left[\omega+\epsilon_{1(2)}(\mathbf{k})\right]}{\left[\omega^{2}-\epsilon_{1(2)}(\mathbf{k})^{2}-\Delta(\mathbf{k})^{2}+i \eta\right]\left[\omega^{2}-\epsilon_{1(2)}(\mathbf{k}+\mathbf{q})^{2}-\Delta(\mathbf{k}+\mathbf{q})^{2}+i \eta\right]}\left(\chi_{\mathbf{k}}+\chi_{\mathbf{k}+\mathbf{q}}\right), \\
& Q_{1(2)}=\frac{\Delta(\mathbf{k})\left[\omega+\epsilon_{1(2)}(\mathbf{k}+\mathbf{q})\right]+\Delta(\mathbf{k}+\mathbf{q})\left[\omega+\epsilon_{2(1)}(\mathbf{k})\right]}{\left[\omega^{2}-\epsilon_{1(2)}(\mathbf{k})^{2}-\Delta(\mathbf{k})^{2}+i \eta\right]\left[\omega^{2}-\epsilon_{2(1)}(\mathbf{k}+\mathbf{q})^{2}-\Delta(\mathbf{k}+\mathbf{q})^{2}+i \eta\right]}\left(\chi_{\mathbf{k}}+\chi_{\mathbf{k}+\mathbf{q}}\right),
\end{aligned}
$$

where the differences arise from the different matrix structure of the potential ( $\tau_{1}$ in this case as opposed to $\tau_{3}$ in the previous case). Again, when we tune the energy to obtain $\epsilon_{i}(k) \sim 0$ the expressions simplify to

$$
\begin{aligned}
P_{i}(\mathbf{k}, \mathbf{q}) & \approx Q_{i}(\mathbf{k}, \mathbf{q}) \\
& \approx \frac{\omega[\Delta(\mathbf{k})+\Delta(\mathbf{k}+\mathbf{q})]^{2} / \Delta_{0}}{\left[\omega^{2}-\Delta(\mathbf{k})^{2}+i \eta\right]\left[\omega^{2}-\Delta(\mathbf{k}+\mathbf{q})^{2}+i \eta\right]} .
\end{aligned}
$$

In this case the observed quantity will be an even function of the bias voltage, $\omega$ and this in principle can be used to distinguish between the two sources of scattering. In Eq. (6) we integrate over the Brillouin zone (BZ) such that $\mathbf{k}$ and $\mathbf{k}+\mathbf{q}$ go over all pairs of states that are separated by momentum $\mathbf{q}$. The main contribution to this sum comes from the vicinity of the contours of constant energy such as the beginning and end points of the arrows in Fig. 1. This means that the intensity of the FT LDOS features in momentum q depend on the gap function at these two points. It is obvious from Eq. (18) that when $\mathbf{q}$ connects two points on the same pocket the vortex-induced scattering will be large and will be added to the impurity scattering (it may enhance or suppress it depending on the relative signs of $V_{0}$ and $\delta \Delta$ and the sign of the bias voltage). On the other hand, if $\mathbf{q}$ connects two points on different pockets the contribution will vanish if $\Delta\left(\mathbf{k}_{i}\right) \approx$ $-\Delta\left(\mathbf{k}_{f}\right)$ as expected in the $s_{ \pm}$scenario.

This is the main finding of this paper-if the OP of the FeSC is of the $s_{ \pm}$structure and has roughly the same magnitude on both the electron and hole pockets the application of magnetic field will affect only features at momenta $\mathbf{q}$ which correspond to intrapocket transitions. Interpocket transitions will stay intact. To best see this effect the bias voltage should be tuned to $\pm \Delta_{0}$ and the odd and even components of the LDOS should be separated.

\section{B. Full lattice model results}

In order to account for both on-shell and off-shell states in an exact fashion, we performed a numerical study of Eq. (12). A sample of our results in the Brillouin zone is presented as a gray scale plot in Fig. 4 and in cuts along the $\hat{x}$

axis in Fig. 3. We identify the transitions $\mathbf{q}_{i}$ as labeled in Fig. 2 by their momentum transfer. In order to ascertain that the transitions are correctly identified we vary parameters (such as the band parameters, the chemical potential, and the energy) and follow the transitions' evolution in momentum space.

In Fig. 3(a), it is clear that while sharp peaks appear in the LDOS at the interpocket momenta $\mathbf{q}_{1}$ and $\mathbf{q}_{2}$ in the case of a nonmagnetic impurity, they are absent in the case of a vortex scatterer. For comparison, when replacing the $s_{ \pm}$OP by a function without a sign change (absolute value) the interpocket transitions appear in both types of scattering.

\section{DISCUSSION}

We have shown that an order parameter which changes sign between the hole and electron pockets of the FeSCs (such as the proposed $s_{ \pm}$) could be distinguished from an order parameter which does not change sign (a simple or anisotropic $s$ wave). We propose to measure the modulations in the LDOS due to scattering off impurities and vortices. The conclusion of our analysis is that the features of the

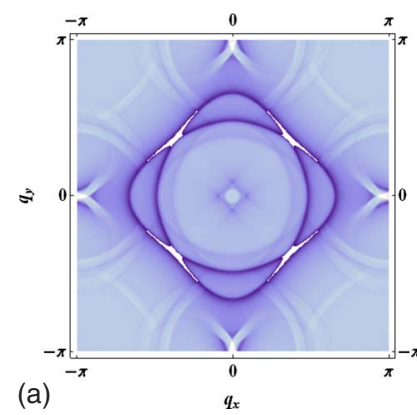

FIG. 4. (Color online) Quasiparticle interference patterns in the Brillouin zone. $\delta n(\mathbf{q})$, Eqs. (10) and (12)-(17), for $\mu=1.5$ and $\omega$ $=0.105$ on a $400 \times 400$ lattice. Left: nonmagnetic impurity-induced interference patterns, Right: vortex-induced interference patterns. The patterns are similar except for the features close to $( \pm \pi, 0)$ and $(0, \pm \pi)$ where the interpocket transitions reside. It is clear that these transitions are missing from the modulations generated by the vortex. 
vortex-induced scattering are sensitive to the OP magnitude and sign. In particular, when the energy is tuned to the gap edge, the intensity of the LDOS features at momentum $\mathbf{q}$ are roughly proportional to $\left[\Delta\left(\mathbf{k}_{i}\right)+\Delta\left(\mathbf{k}_{f}\right)\right]^{2}$, where $\mathbf{k}_{i}$ and $\mathbf{k}_{f}$ are two points on the relevant contours of constant energy which are separated by momentum q. This means that if the OP is of similar magnitude but opposite sign on the two points a cancellation will occur. Such cancellation is expected for the $s_{ \pm}$OP but not for an $s$ wave and is therefore a signature of the $s_{ \pm}$OP. It is important to emphasize that the conclusion of the analysis presented in this work is quite robust. Numerical simulations with modified lattice parameters and chemical potential have yielded modified quasiparticle interference patterns. However, the absence of the transitions $\mathbf{q}_{1}$ and $\mathbf{q}_{2}$ appears in the vortex scattering contribution whenever the OP changes sign between the electron and hole pockets.

We would like to mention that the Born approximation, chosen for the current study, is both simple and appropriate when the scattering potential is weak. For stronger scattering the $t$ matrix may be of use. Its strength is generally in finding impurity bound states $^{33}$ or vortex bound states (Caroli-de Gennes-Matricon). ${ }^{34}$ In general, it amounts to replacing the bare scattering matrix by an energy-dependent one. Often, the $t$ matrix does not add any momentum dependence and therefore will lead to the same quasiparticle interference patterns as the Born approximation.

The proposed experiment is scanning tunneling spectroscopy in magnetic field. Such measurements have been successfully carried out in the cuprates ${ }^{35}$ and have been interpreted in a similar fashion to that proposed here. ${ }^{25}$ We are hopeful that advances in material synthesis will allow similar studies of the FeSCs. Their quasi-two-dimensional structure is ideal for scanning tunnel microscope and their transition temperature is high enough for such experiments. An important consideration is the vortex core size in FeSC. In general, the larger the core size, the harder it is to detect features at large momenta (close to the BZ edges). In the Born approximation, in order to replace the pointlike vortex considered here by a more realistic one, the perturbation $\delta \Delta$ should be replaced by a more smoothly varying function in real space with a characteristic length scale $\xi$, the coherence length. Its Fourier transform $\delta \Delta(\mathbf{q})$ will multiply our result for $\delta n(\mathbf{q}, \omega)$ creating an envelope beyond which features are suppressed. In $\mathrm{BaFe}_{1.8} \mathrm{Co}_{0.2} \mathrm{As}_{2}$ the coherence length was measured to be $\sim 27.6 \AA{ }^{36}$ which may lead to a significant signal suppression at $(\pi, 0)$. However, this coherence length is similar to that of BiSCCO (a member of the cuprate family) ${ }^{37}$ where FT LDOS features were seen clearly even at the edges of the Brillouin zone. ${ }^{26}$ This suggests that the vortex size and structure in $\mathrm{BaFe}_{1.8} \mathrm{Co}_{0.2} \mathrm{As}_{2}$ may be suitable for the suggested experiment.

\section{ACKNOWLEDGMENTS}

The authors would like to acknowledge useful discussions with M. Franz, O. Motrunich, and Z. Tešanović, the hospitality of the Aspen Center for Physics where some of the work has taken place, and funding from the Caltech SURF program (E.P.); the Packard and Sloan Foundations, the Institute for Quantum Information under NSF Grants No. PHY0456720 and No. PHY-0803371, and the Research Corporation Cottrell Scholars program (G.R.).
${ }^{1}$ Y. Kamihara, T. Watanabe, M. Hirano, and H. Hosono, J. Am. Chem. Soc. 130, 3296 (2008).

${ }^{2}$ J. Bardeen, L. N. Cooper, and J. R. Schrieffer, Phys. Rev. 108, 1175 (1957).

${ }^{3}$ C. C. Tsuei and J. R. Kirtley, Phys. Rev. Lett. 85, 182 (2000).

${ }^{4}$ W. N. Hardy, D. A. Bonn, D. C. Morgan, R. Liang, and K. Zhang, Phys. Rev. Lett. 70, 3999 (1993).

${ }^{5}$ Y. Nakai, K. Ishida, Y. Kamihara, M. Hirando, and H. Hosono, J. Phys. Soc. Jpn. 77, 073701 (2008).

${ }^{6}$ H.-J. Grafe, D. Paar, G. Lang, N. J. Curro, G. Behr, J. Werner, J. Hamann-Borrero, C. Hess, N. Leps, R. Klingeler, and B. Büchner, Phys. Rev. Lett. 101, 047003 (2008).

${ }^{7}$ K. Matano, Z. A. Ren, X. L. Dong, L. L. Sun, Z. X. Zhao, and G. Zheng, EPL 83, 57001 (2008).

${ }^{8}$ H. Mukuda, N. Terasaki, H. Kinouchi, M. Yashima, Y. Kitaoka, S. Suzuki, S. Miyasaka, S. Tajima, K. Miyazawa, P. Shirage, H. Kito, H. Eisaki, and A. Iyo, J. Phys. Soc. Jpn. 77, 093704 (2008).

${ }^{9}$ J. D. Fletcher, A. Serafin, L. Malone, J. G. Analytis, J.-H. Chu, A. S. Erickson, I. R. Fisher, and A. Carrington, Phys. Rev. Lett. 102, 147001 (2009).

${ }^{10}$ S. Kawasaki, K. Shimada, G. F. Chen, J. L. Luo, N. L. Wang, and G. Q. Zheng, Phys. Rev. B 78, 220506(R) (2008).

${ }^{11}$ H. Ding, P. Richard, K. Nakayama, K. Sugawara, T. Arakane, Y.
Sekiba, A. Takayama, S. Souma, T. Sato, T. Takahashi, Z. Wang, X. Dai, Z. Fang, G. F. Chen, J. L. Luo, and N. L. Wang, EPL 83, 47001 (2008).

${ }^{12}$ T. Kondo, A. F. Santander-Syro, O. Copie, C. Liu, M. E. Tillman, E. D. Mun, J. Schmalian, S. L. Bud'ko, M. A. Tanatar, P. C. Canfield, and A. Kaminski, Phys. Rev. Lett. 101, 147003 (2008).

${ }^{13}$ L. Zhao, H.-Y. Liu, W.-T. Zhang, J.-Q. Meng, X.-W. Jia, G.-D. Liu, X.-L. Dong, G.-F. Chen, J.-L. Luo, N.-L. Wang, W. Lu, G.-L. Wang, Y. Zhou, Y. Zhu, X.-Y. Wang, Z.-Y. Xu, C.-T. Chen, and X.-J. Zhou, Chin. Phys. Lett. 25, 4402 (2008).

${ }^{14}$ K. Hashimoto, T. Shibauchi, T. Kato, K. Ikada, R. Okazaki, H. Shishido, M. Ishikado, H. Kito, A. Iyo, H. Eisaki, S. Shamoto, and Y. Matsuda, Phys. Rev. Lett. 102, 017002 (2009).

${ }^{15}$ L. Malone, J. D. Fletcher, A. Serafin, A. Carrington, N. D. Zhigadlo, Z. Bukowski, S. Katrych, and J. Karpinski, Phys. Rev. B 79, 140501(R) (2009).

${ }^{16}$ C. W. Hicks, T. M. Lippman, M. E. Huber, J. G. Analytis, J. H. Chu, A. S. Erickson, I. R. Fisher, and K. A. Moler, Phys. Rev. Lett. 103, 127003 (2009).

${ }^{17}$ S. Raghu, X.-L. Qi, C.-X. Liu, D. J. Scalapino and S.-C. Zhang, Phys. Rev. B 77, 220503(R) (2008).

${ }^{18}$ I. I. Mazin, D. J. Singh, M. D. Johannes, and M. H. Du, Phys. Rev. Lett. 101, 057003 (2008). 
${ }^{19}$ K. Seo, B. A. Bernevig, and J. Hu, Phys. Rev. Lett. 101, 206404 (2008).

${ }^{20}$ R. Arita and H. Ikeda, J. Phys. Soc. Jpn. 78, 113707 (2009).

${ }^{21}$ V. Cvetkovic and Z. Tešanović, Phys. Rev. B 80, 024512 (2009).

${ }^{22}$ F. Wang, H. Zhai, Y. Ran, A. Vishwanath, and D.-H. Lee, Phys. Rev. Lett. 102, 047005 (2009).

${ }^{23}$ D. Parker and I. I. Mazin, Phys. Rev. Lett. 102, 227007 (2009).

${ }^{24}$ J. Wu and P. Phillips, Phys. Rev. B 79, 092502 (2009).

${ }^{25}$ T. Pereg-Barnea and M. Franz, Phys. Rev. B 78, 020509 (2008).

${ }^{26}$ J. E. Hoffman, K. McElroy, D.-H. Lee, K. M. Lang, H. Eisaki, S. Uchida, and J. C. Davis, Science 297, 1148 (2002).

${ }^{27}$ See, for example, T. Pereg-Barnea and A. H. MacDonald, Phys. Rev. B 78, 014201 (2008).

${ }^{28}$ T. Pereg-Barnea and M. Franz, Phys. Rev. B 68, 180506(R) (2003).

${ }^{29}$ Y.-Y. Zhang, C. Fang, X. Zhou, K. Seo, W.-F. Tsai, B. A. Bernevig, and J. Hu, Phys. Rev. B 80, 094528 (2009).
${ }^{30}$ T. Pereg-Barnea and M. Franz, Int. J. Mod. Phys. B 19, 731 (2005).

${ }^{31}$ L. Capriotti, D. J. Scalapino, and R. D. Sedgewick, Phys. Rev. B 68, 014508 (2003).

${ }^{32}$ A. V. Balatsky, M. I. Salkola, and A. Rosengren, Phys. Rev. B 51, 15547 (1995).

${ }^{33}$ W.-F. Tsai, Y.-Y. Zhang, C. Fang, and J. Hu, Phys. Rev. B 80, 064513 (2009).

${ }^{34}$ Y. Nagai and Y. Kato, eprint arXiv:0908.4451 (unpublished).

${ }^{35}$ T. Hanaguri, Y. Kohsaka, M. Ono, M. Maltseva, P. Coleman, I. Yamada, M. Azuma, M. Takano, K. Ohishi, and H. Takagi, Science 323, 923 (2009).

${ }^{36}$ Y. Yin, M. Zech, T. L. Williams, X. F. Wang, G. Wu, X. H. Chen, and J. E. Hoffman, Phys. Rev. Lett. 102, 097002 (2009).

${ }^{37}$ S. H. Pan, E. W. Hudson, A. K. Gupta, K.-W. Ng, H. Eisaki, S. Uchida, and J. C. Davis, Phys. Rev. Lett. 85, 1536 (2000). 